\title{
AVUNET Author: An Authoring System for Distance Learning Platform
}

\author{
${ }^{1}$ Lamri Douidi, ${ }^{2}$ Mahieddine Djoudi, ${ }^{1}$ Chabane Khentout \\ ${ }^{1}$ Department of computer science, University Ferhat Abbas of Setif, Algeria \\ ${ }^{2}$ Laboratoire SIC et équipe IRMA, UFR Sciences SP2MI Université de Poitiers Téléport 2, \\ BP 3017986960 Futuroscope Cedex, France
}

\begin{abstract}
An authoring system was presented in this study, "AVUNET Author" which allows the creation of interactive learning object within a distance teaching platform on the Internet. For the conception of this system, we stated with the assumption that the content of distance teaching is not just to put online the content used for classroom teaching. In addition to the classical functions such as editing and formatting a text, the system is enriched with functions to cater for the needs of the instructor designing the course content. The users can thus generate automatically the table of contents, create questions for evaluation purpose and manage the bibliographical references. The graphical construction of the content allows an easy manipulation and a better course structure for a later adaptation to the learner's profiles. We started experimenting with the system at the University of Sétif in collaboration with a group of computer science instructors.
\end{abstract}

Key words: Authoring system, distance education, content design, learner evaluation

\section{INTRODUCTION}

The content is defined as a set of structured teaching and learning experiments (content objectives, skills objectives, specific objectives, different routes and progression rules, teaching and training activities, etc.) planned and offered under the direction of an educational institution in order to achieve predetermined educational goals. It thus includes the course itself (text, images, animated images, sounds and video, self evaluation exercises and recommendations) and training scenarios designed by the teacher for the intended learner ${ }^{[1,2]}$.

In the domain of distance learning, the content development is an important stage of the design of teaching process. Indeed, the content is often regarded as an input parameter to an educational system. We consider that the contents, while focusing on knowledge relative to a given subject, must also be concerned with didactic resources necessary for acquisition, by the learner, of this knowledge in teaching and learning situations ${ }^{[2]}$. Indeed on line course requires a very specific scenario and a structured presentation. Once that information is accessible on Internet, it does not necessarily mean that we have obtained a training system if we did not think about the way in which people will access the information and what they will use it for. It is important to clearly represent the information and knowledge on the structural level and to think and decide which strategy and diffusion means to use.

Interactions quality is very important in the teaching process, requiring powerful and active scenarios of communication for the learner. Sometimes this requirement for quality imposes on the teachers a complete recasting of their courses and the manner in which they design them. This raises the need to propose authoring systems for distance teaching.

\section{AUTHORING SYSTEMS PRINCIPLES}

An Authoring System is a computer tool, which makes it possible for the users to create, modify and delete training material. The essential goal is to make it possible for the end-users to manipulate the content. It is a system that helps people who are not information technology specialists to develop teaching modules in a convenient and fast way. For example books or catalogues of product on CD-ROM, of information or training supports in an Intranet, etc. It includes:

* Learning methodology definition

* Information modularity

* Objective based approach

* Multimedia objects development

Many authoring systems propose either a compatibility with HTML environment, or an extension allowing the applications deployment on the network. The following characteristics are expected in an Authoring System:

* Accessibility to non-programmers

* Possibility of structuring the presentation: linear structure, hierarchical, topic, star

* Implementation of various resources: image, text, sound, video

Corresponding Author: Lamri Douidi, Département d'informatique, Université Ferhat Abbas, 19000 Sétif, Algérie 
* Interactivity

* Updated easily via a local area network or Internet

An authoring system has a set of tools that allow the user on one hand to be able to import, to manipulate or produce the various types of information (textual, visual or sound) to be included in hyper-document (for example, tools for graphic creation, animation and assembly). On the other hand, it gives the user the possibility of designing the hyper-document structure (nodes and links) and its graphical interface (node format, types of buttons controlling the connections, etc.). The authoring systems may or may not offer the hyper-document author the possibility to use a programming language to define different hyperdocument functions.

During this last decade, several works concentrated on the design and implementation of ITS authoring systems. Tom Murray listed more than two dozen of references in his recent study. Among these systems we mention here as examples: CREAM-tools, Eon, Iris, Training Express, etc. ${ }^{[3]}$. These systems are classified in seven categories according to the type of ITSs they produce. These categories are: curriculum sequencing and planning, tutoring strategies, device simulation and equipment training, domain expert system, multiple knowledge types, special purpose and intelligent/adaptive hypermedia.

Given that ITSs are often described as having four main components (domain module, tutoring module, student model and student interface), the authoring systems must therefore theoretically include all the necessary tools allowing to construct these components. Nevertheless, it has to be recognized that, very few systems require from the author to construct every thing needed. The other systems are limited usually to tools for constructing one, two or at limit three components among the four. The remaining components are generally predefined in a pattern of ITS and the author is solicited only to introduce necessary parameters for their functionality ${ }^{[4]}$.

\section{COURSE MANAGEMENT SYSTEMS}

The distinctive characteristic of course management systems is to make it possible for individual instructors who have little or no knowledge of HTML or other web oriented programming languages to design and offer online educational contents. The development tools should be integrated in an environment, which will allow the instructors to create Web pages, upload documents and create on line tests and questionnaires and to add components like electronic mail and discussion forums. These systems should also contain management tools that can be used for students' registration and necessary follow-up. Usually, course management systems are designed for educational institutions and offer reduced costs similar integrated tools for content creation. They offer generally various levels of student's record management and follow-up of their progress. They seldom support a third party tools. There are a number of course management systems available but very few are dedicated specifically to be integrated in a distance teaching platform. These systems differ from each other due to the nature (course, evaluation materials and the learner selfevaluation) and the content representation (summary, tree, graph, etc.). The majority of these systems use either a format that requires a client (client software to allow the learner access to the content) or propose procedures to export the HTML format. Teaching platforms via the Web integrate an authoring system for content creation and management within the system. These systems do not include the bibliographical references management. In the following we will present some well known systems. A complete list of course management systems in open source can be found in $\mathrm{CMS}^{[5]}$.

Serpolet and cognifer: SERPOLET (Educational and Training system) of A6-MediaGuide Company is a complete and adaptable platform to organize, plan, learn, follow up and manage. It is made up of course editors, sequences, hypertext and multi-media. It uses a synchronous and asynchronous teaching approach. It works under Windows NT/95/98, DOS, UNIX and OS/2 environments. In addition A6-Media Guides Company developed COGNIFER which is a platform for university education. This platform which works with SERPOLET, allows the development, organization, planning, training, follow-up and the administration of a particular program.

Serpolet and cognifer are designed for professionals in teaching and communication who are able to integrate, with method, all expertise in the field: teaching, didactic, media ${ }^{[6,7]}$.

Blackboard learning system: Blackboard Learning System allows the educational institutions, companies and commercial or governmental educational services suppliers to offer administrative services, of community or another on line educational service. Blackboard course management system offers tools for course content creation, a robust evaluation engine, synchronous and asynchronous tools for collaboration and a set of commands for the instructors. Blackboard Building BlocksSM module proposes an open architecture allowing training applications, the interfaces and the system services to interact without discontinuity with the Blackboard platform. The existing tools Building Block allow for example content management, wireless communication and functionality.

Moreover, Blackboard pledges the accessibility of Eeducation platforms. We are using the best tools in the 
accessibility field to grant our software the standards regulation regarding accessibility ${ }^{[8]}$.

WebCT: WebCT (Web Course Tools) is one of the most used course management systems on Internet in higher education everywhere in the world because its interface allows the use of more than ten different languages. It requires a small investment and it is simple to install and use. WebCT makes it possible to create courses organized in a hierarchical way. It offers course templates very easy to modify and allows the inclusion of animated videos, sound, tests and glossaries creation.

The interface gives access to a complete map of the course which makes it possible to visualize or modify the course. It also allows the management of information about the students. WebCT allows the user to adapt the appearance of the interface according to each course. There are many tools available for the student to organize his/her documents, for assistances, to publish his/her work and for self-evaluation. This platform envisages three roles: administrator, course creator (teacher) and student. WebCT supports on line course, makes it interactive by adding to it, in a structured and contextualized way, communication and evaluation activities as well as a certain number of tools which enrich the student's workspace. The media used are all the kind supported by Internet standard protocols and the existing navigators. For 3 media, texts, fixed or animated images, tests, WebCT either creates or imports the teaching materials ${ }^{[9]}$.

Claroline: Claroline (classroom online) allows the instructor to design, manage and create their courses through the Web. It makes it possible for the students to follow these courses and to participate in discussion concerning these courses. The platform has tools for forum discussion, work publication, calendar, documents sharing, links management, quiz, etc.

Claroline is a dynamic and modular space, allowing a progressive engagement of teaching in a reflective educational approach. Its ease of use allows an immediate appropriation and a fast perception of the benefit of the use of Internet in a course. This speed for getting used to this kind of environment helps to motivate the teachers and often leads them to work more on the method, to even take part, via their advises and their requirements formulation, for Claroline development.

Claroline does not impose the use of complex tools or suggest a particular method. It functions, as far as possible, as a simple support to the instructors' choices according to their discipline requirements, their teaching model and to the public they are addressing. Developed in PHP/MySql, the platform is currently available in several languages. It is an open-source software, thus its use is completely free.
Ganesha: Ganesha is a simple platform that concentrates on the education aspect and does not require deep knowledge of the Internet. The training module consists of files accessible via Internet. These files include: course support, multiple-choice questionnaires (QCM), tests, animations, simulations and a data management system that keeps track of students' information such as scores, etc.

During training sessions, trainees and trainers use collaborative tools. The session alternates between work on Internet and preparation work. The distance teaching platform is the software tool which creates and integrates the modules and manages the sessions.

The courses exist in various formats (Excel, PowerPoint and Word documents) and are used as they are, on the basis of the principle that the Net surfers will print them.

Course complements include all what Internet can bring in term of dynamic explanation, interactive and multimedia. The objective is to develop course complements. Tests are designed and used to check the course understanding.

The emphasis is on the asynchronous tools which do not introduce a time constraint. The tutor must be able to consult the group activity, to intervene in the discussion forum, to answer e-mail and to manage a planning for the courses need.

The platform is open, dynamic, simple and accessible via a standard computer and an internet connection. Ganesha is an open-source platform developed in PHP/Mysql and it is available for free use ${ }^{[10]}$.

Cose: Cose is a virtual training environment designed and implemented by "Staffordshire University Enterprises Ltd". It has two interfaces: an interface dedicated to the instructors so that they can create their course and an interface dedicated to the students so that they can access these courses easily. It provides tools for educational environment creation and gives the possibility for the environment to be developed in a coherent manner, i.e. which allows a good practice. Using this tool the user can exploit a broad range of media ${ }^{[11]}$.

\section{AVUNET PLATFORM PRESENTATION}

AVUNET (Algerian Virtual University) is a Multilanguage environment (Arabic, English and French) for distance education making use of the new information and communication technology specifically Internet and hypermedia. It has a structure close to that of LearningSpace, TopClass, Librarian or WebCT. Based on client-server architecture, the platform is developed in PHP/MySQL and is software independent. The data set is stored on the server in a centralized database ${ }^{[12]}$.

AVENUT platform contains three systems: 
1. A production authoring system contains the necessary tools for tasks' production. It contains amongst other things a content design environment and an evaluation space to improve the learner knowledge and abilities. We detail the system functionality in sections 5 to 8 .

2. A communication and management server made up of several modules. An information module which contains the various files and data needed by the user's teaching or training activities. A co-operative and communication module which has the means to make it possible for users to interact with each other, to accomplish team works or to take part in discussions. In order to favor the co-operative learning, the interfaces are conceived in such a way to make the presence of the others known by providing indication of their availability and their remarks on the teaching material.

3. A help system which makes it possible for the learner to obtain assistance or advice or an adaptation of the environment from the computer system. The objective of the designed system is to give the learner the possibility to locate him/herself with respect to time and space during a training session. The learner is presented with a chart of courses and visited pages, thus enabling him/her to have an explicit representation of the virtual space. Various visualization levels are set up in order to make the chart more visible and not overloaded. A temporal panel is displayed permanently allowing the learner to monitor and optimize the training time. The user has also the possibility to access online help and a glossary containing the terms frequently found on Internet and likely to be misunderstood by beginner. The system gives access to a set of tools: notebook, diary, work plan, etc. $^{[13,14]}$.

\section{AVUNET AUTHORING SYSTEM}

Objectives and content design approach: Our objective is to design and implement an authoring system for content design in a format directly accessible by the learner. It is a matter of proposing a representation model for the content related to the topic being taught, taking into account the perspectives of educational domain and pedagogic. Our idea is to create and organize an online access environment to a rich and varied content, which can support course development, planning and implementation teaching and some aspects related to learner modeling.

Our contents design approach supports three prospects for topic organization: domain, pedagogy and didactic. In this approach the domain aspect is achieved by a model, which represents and organizes the domain knowledge based on the existing logical links. The organization of the objectives consists in particular in modeling the necessary preconditions to their realization and studying the impact that an objective can have on domain knowledge. Didactic dimension amounts to producing a model that defines and organizes the different tactical means necessary to the teaching of the considered topic.

Pedagogic and graphical map definition: The quality of a teaching platform on Internet is based mainly on the information (textual and/or graphic) organization, the navigation flexibility and the interactivity. The design of a teaching platform on Internet supposes the analysis of the needs and the site relevance. The needs' analysis will help in answering the following questions:

* What are the needs of the educational platform: interactivity, exercises, simulations, information retrieval, data processing, etc?

* Why use the Internet: distance learning, computerassisted co-operative work, interactive learning, pedagogic advising, etc?

* Who are concerned: academicians, distance learners, training for companies, general public, etc?

* What are the technical considerations to take into account: computer power, screen type and size, multi-media, Internet network, etc?

* And finally how is the time distributed on the project?

The teaching design consists in: defining the goals and the objectives, structuring the contents, setting the teaching strategies, designing the interface parameters, building the flow chart, designing the screen pages models and writing the content of the screen pages $[14,15,16]$.

System architecture: AVENUT Authoring system allows an instructor to design a pedagogical content for distance education within the virtual University. The goal of this system is to allow the creation of courses that can be used by different instructors. Each instructor can personalize his/her course based on the objectives and problems related to the concerned course. For that purpose it is recommended to decompose the material in learning objects that are independent from each others as much as possible. Each instructor can create his/her course by choosing from a database of learning objects the ones that respond to the course's objectives and problems. These learning objects are organized in such a way to create a coherent network. 


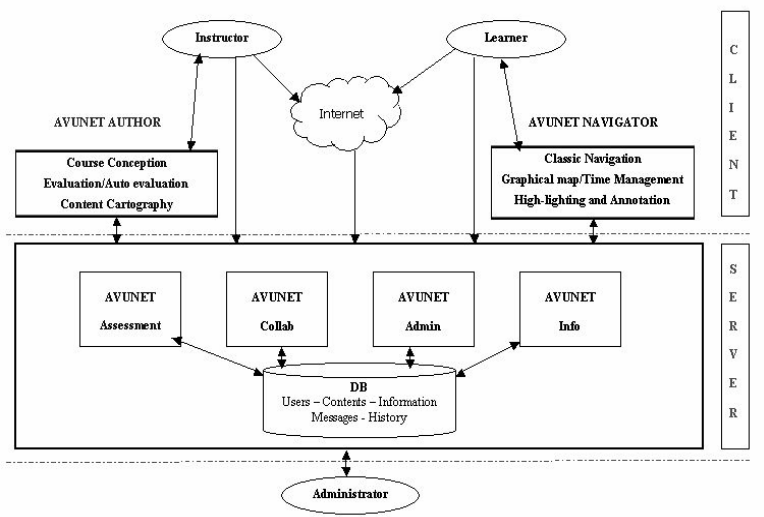

Fig. 1: AVUNET Author within the platform
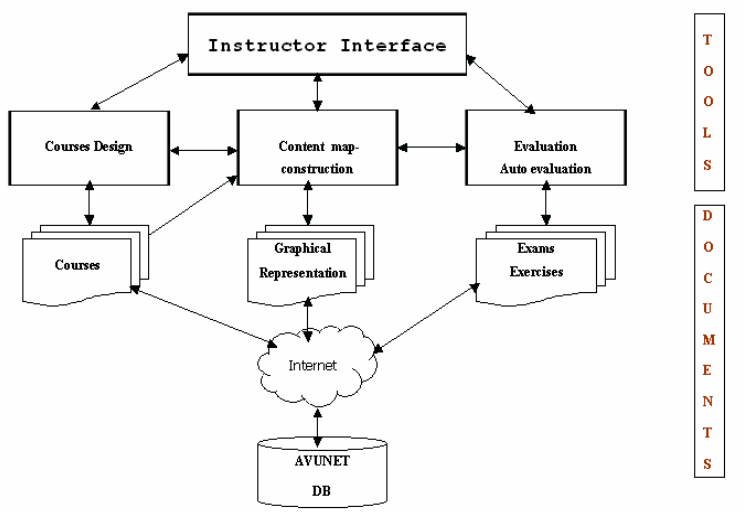

Fig. 2: AVUNET Author simplified architecture

\section{COURSE DESIGN}

Course Design Interface allows the integration objects of different types (such as images, equations, diagrams, questionnaires ...) and an organization based on several criteria (chapter, detail level). Moreover it will manage various facilities of path to follow: synopsis, index and glossary. These points would allow the students to take a nonlinear lecture adapted to their needs. They can check the knowledge obtained by solving exercises.

The course composition is made easy by the fact that each portion of the course (exercise, theoretical part, examples) is independent of the linear structure of the document. We thus decompose the course in distinct entities and design objects from the given data. We adopted a three level hierarchical model to represent a course document:

* The basic elements are the objects that we insert: images, diagrams, equations, texts, etc. They cannot contain any other object.

* The first type of composite objects can contain only basic objects. Such an object can for example be a theorem, an example, a exercise, etc
* The second type of composite objects can contain all the types of objects: basic and composite objects. It could be the entire course, a chapter, a subsection, or an exercise sheet.

Course design module allow the development of the course on line, with all qualities of text processing and Web sites editor. One will be able in particular to insert various elementary objects, such equation, diagrams, links, images, etc. Moreover one will be able to visualize an outline of the generated course. Models in the forms of style sheets developped according to the teaching plan are available to work the course details.

\section{LEARNER EVALUATION}

Evaluation role within the system: The evaluation is a fundamental aspect and impossible to circumvent in education. It is indeed crucial that the instructor can evaluate what the students have understood and what they did not. It is also important for the students, during their training, to be able to evaluate their knowledge. There are several ways on how to evaluate a learner. The most used evaluation types are diagnosis evaluation, the formative evaluation, the training evaluation and the summative evaluation.

The function of evaluation has an important role within the framework of the training method proposed by the authoring system. In addition to the traditional roles of any evaluation tool, the authoring system evaluation function must make it possible for the learner to distinguish the concepts already learned from those not learned yet. Consequently the facility of spreading out the learning process over several periods of time must be available ${ }^{[6]}$.

This evaluation thus has double objectives. One it must make it possible for the instructor to propose, at the start of each learning object, either a pre-test, or a test of pre-requisites. Second it must also make it possible for learners to self- evaluate at the end of each learning object by proposing an exit test.

The various types of possible questions in the evaluation module are as follows: question with simple answer, question with multiple choices, fill in the missing text, make correspondence between two lists, or order a given set of elements.

Actually the self-evaluation has a very important role within the system since it is the learner who decides to be evaluated or not. The instructor makes at the disposal of the learner a questionnaire allowing him/her to check the progress. The evaluation is an integral part of the learning process. Moreover, it is the learner who decides by him/herself whether the obtained result in the evaluation is sufficient to consider the material has been understood. 
Questionnaire design for learner evaluation: In AVUNET authoring system, we implemented a module for learner's evaluation and self-evaluation. Only the instructor in charge of the course can access this module in design mode. Each questionnaire is associated with a learning object. In this mode, the user can create a new questionnaire or open an existing questionnaire. The user must be able to choose whether the questionnaire is intended for the general evaluation or the self-evaluation (should the response time be fixed or not). He/she has the possibility of choosing the grading system for each question and for the whole questionnaire (number of correct answers, percentage, mark out 20, etc.). It is possible to illustrate the question by a text, image and possibly an audio or video file. A feedback is associated to each response in the form of a detailed comment.

Once the questionnaire is finished, it is saved on the platform server. The learner can access it via the web navigator or learner interface. The user chooses the questionnaire of the concerned subject. Based on the questionnaire the learner can either take a general evaluation or a self-evaluation. In self-evaluation mode, the user has the choice between having the questions (and even the answers) in order or in a random order. $\mathrm{He} / \mathrm{she}$ must be able to choose between displaying the answers instantaneous or wait until the end. The evaluation process is done while moving forward from one question to another with the possibility of returning backward. At the end of the questionnaire, in selfevaluation mode the grade as well as the correct answers and feed back are displayed. In evaluation mode, the results are recorded on the server and/or sent by email to the concerned instructor.

We have designed and implemented a tool to handle the answers given by learners during the automatic evaluation. This tool is used also to analyze the grades of group learners: display learners' lists and their grades, compute the averages, maximum and minimum grade, etc. This option gives the possibility to the learner to compare him/herself to other users automatically. This comparison is also an interesting way for the user to see where he/she stands comparatively to others ${ }^{[12]}$.

\section{IMPLEMENTATION}

AVUNET Authoring System is a client tool designed and implemented in DELPHI under window platform using object oriented techniques. We adapted a progressive approach in integrating functions offered to the instructor through a simple graphical interface. Because the kernel is independent from the interface the system can be adapted very easily to the instructor's requirements.

In the current version of the system the course creation and learner evaluation modules are working properly.
The instructor can use AVUNET Author to design a course based on a preset graphical and pedagogical chart. The summary (or table of content) is generated automatically as the instructor is inserting the material. The content classical manipulations are available through the graphical interface bar tools.

The learner evaluation module allows the design of questionnaires for evaluation and self-evaluation. The questionnaires are saved on the platform server to be used in the learner mode. Also the system can generate the pedagogical bag for the learner. We are working on the implementation and integration of the remaining modules (references management, annotation and marking and graphical manipulation of the content).

\section{SYSTEM EVALUATION}

Experiment protocol: The experimentation is an important phase of the prototype implementation, since it enables us to validate the view that we have of the system. We have started testing the current version of the system in real situation within the teaching activities of the instructors. A limited number of instructors (Department of Computer Science, University of Ferhat Abbas (Setif, Algeria)) have participated in the experiment. The essential objective of this study is to find out whether the use of this system has any effect on the perception that instructors have concerning the construction of pedagogical content for online teaching. Also we would like to know what the users' interest level is in our system.

In order to emphasize the principal characteristics of the system, we selected several tasks which we wanted the instructors to do during their evaluation session. We prepared a list of the tasks defined according to a scenario, which we gave to each user at the beginning of the evaluation. We also prepared a sheet of instructions for the person charged to follow-up the evaluation procedure.This person would explain explain informally this sheet to the users to guide to accomplish the assigned tasks. We observed the instructors during an evaluation session and documented their reactions. At the end of the session, each user filled a questionnaire related to the system. We analyzed these questionnaires to find out what needs to be improved.

Our working assumptiom was the use of the system could be a motivating element for the instructors. Since this syetem would allow them to create in an easy way publishable courses on the web. However, we thought that the use of the system could also generate technical complications for Instructors that do not have experience in the use of computers and different software.

Evaluation questionnaire: The system usefullness was measured by questionnaires that were filled by instructors after they have used the system to 
create teaching material for a real example to support a course. Two questionnaires were used: the first one is used to collect information about the instructors and the second one is used to evaluate the system.

Instructor questionnaire: In order to collect some information about the instructors, we asked them some questions which relate primarily to their previous experience in course design and creation. Here are some sample questions extracted the evaluation questionnaire.

* What is your function or title?

* What subjects do you teach?

* Under which environment do you work (Windows, MacOS, Linux or others)?

* Which software do you currently use to deliver your courses and/or supporting material for your courses (Word, PowerPoint, etc.)?

* Would you be willing to train on a tool that helps to design and deliver online courses?

* Did you deliver or are you currently delivering online courses?

* If yes, which tools do you use to design and create your online courses (FrontPage, DreamWeaver, etc.)?

* What are the main advantages of these tools?

* What are the main disadvantages of these tools?

* Would you be ready to invest some of your valuable time to learn and use another tool?

System questionnaire: After the instructors have used the system, we asked them to fill in an evaluation questionnaire about our system. The users were asked to make the most objective possible assessment on the system. Here are the main questions given in the questionaire:

* What system functions did you test?

* What are the tasks that you were not able to achieve?

* What are the functions that you think must be part of such a system?

* What are the functions that must be added to the system?

* Is it important for you to have the facility to configure the general aspect of the interface?

* Do you prefer to recover your data via cut/paste or drag\&drop?

* Which functions are missing from the interface? Or which functions do you think need to be changed to optimize the use of this system?
* Do you prefer to be guided in your actions or to be completely free when preparing your course presentation?

* What do you think of automatizing the task of reusing parts of an existant course?

* Did you notice any bugs in the interface? If yes, please report them to us?

* Give us any remarks (needs, special attention, any thing that might improve and/or make the envoronment easy to use)?

Evaluation result discussion: The participants showed a real interest in the system where the essential tools, to create and manipulate pedagogical content, are available via a unique interface. They found out that the graphical interface made the system very easy to use. The overall organization of the interface is appropriate for the instructors, both on the level of the window distribution on the screen and on the level of the components (icons or names given to the tools for example). However, some users had some remarks concerning the icons we have used. Indeed, we created ourselves some icons, such as the opening and closing arrows of course object panels, or the icons representing each object. The tasks associated with these icons would have been more intuitive if we had re-used windows standardized icons for example.

The users appreciated the presence of a detailed plan of the course by components in a separate window. However, some of them would have also wished that the architecture of the course be marked visually on the level of the provision of the objects in the course. The current interface indeed makes it possible to define titles for each part. It could be interesting to improve this graphic aspect, while revealing visible containers for each part.

The functionalities which we implemented were also appreciated by the users, in particular the creation of teaching objects. Indeed, the concept of object on which our prototype rests seems a good solution to facilitate course composition. However, some of these functionalities were limited compared to the users' expectation. Indeed, several remarks were raised, in particular on objects management, the export formats proposed and on a possible multi-user version of AVUNET Authoring system.

Many instructors expressed the desire to be able to import data in different formats ( $\mathrm{rtf}$, txt and HTML). The next version of our system will have this possibility. Other instructors would like to have the possibility to export their new courses in other formats (for example PDF which makes the printing easy). They would like also to have the facility to create labs and/or work sheets. 


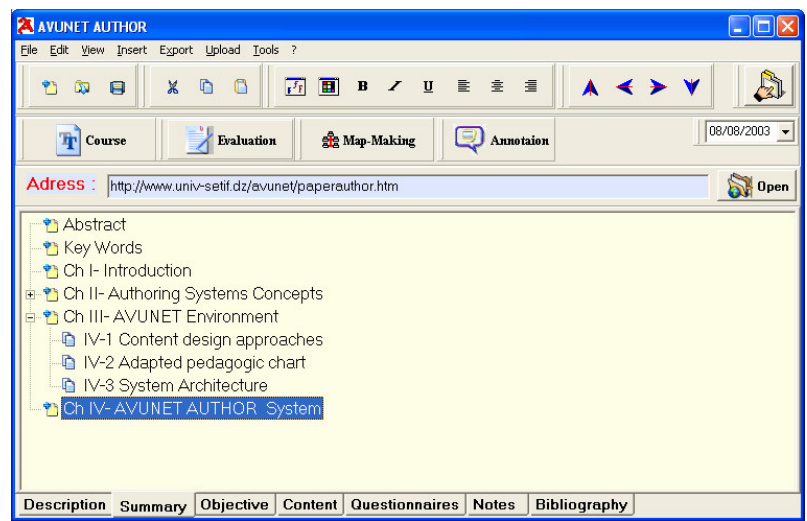

Fig. 3: Interface screen

\section{CONCLUSION}

AVUNET platform interface provides an easy access to the available resources and to the various integrated tools. The instructors can create and deploy teaching material in an easy way using this system. It gives the users the possibility of creating course and related exercises for evaluation or self-evaluation. We have started experimenting with the use of the system in real teaching situation. This experimentation allows us to collect information on the effective activities of the users. We can thus validate or question certain technical choices and determine with more precision the adaptations that have to be made to the integrated tools. We continue the development of AVUNET authoring system and particularly the references management module. We are currently working to see what contribution the XML technology can add to content modeling and structure ${ }^{[17]}$. We are also investigating the possibility of integrating the cooperation and intelligence to the system ${ }^{[4]}$.

\section{REFERENCES}

1. Nkambou, R., Frasson C., Gauthier G., 2003. CREAM-Tools: An Authoring Environment for Knowledge Engineering in Intelligent Tutoring Systems. In: Murray, T., Blessing, S. and Ainsworth, S. (Eds.): Authoring Tools for Advanced Technology Learning Environments: Toward Cost-effective Adaptative, Interactive and Intelligent Educational Software. Kluwer Publishers, pp: 93-138.

2. Dufresne, A., 2001. Conception d'une interface adaptée aux activités de l'éducation à distanceExploraGraph. Revue Sciences et Techniques Éducatives, 8: 3.
3. Murray. T., 1999. Authoring intelligent tutoring systems: An analysis of the state of the art. Intl. J. AI in Education, 10: 98-129.

4. Harous S., Djoudi M., 2003. A cooperative authoring system for intelligent tutoring systems. The Intl. Conf. Internet Computing (IC-IC'2003), Las Vegas, Nevada, USA.

5. CMS., 2005. Open Source Course Management Systems- Oct. 22, http://www.edtechpost.ca/gems/open_source_cms3 .htm, Visited Nov. 2005

6. Claes, G., 2002. La gestion et l'évaluation pédagogique et administrative par le Web. 2eme Colloque international sur l'université virtuelle, Alger.

7. A6, 2005. http://www.a6.fr/ Visited Nov. 2005.

8. Blackboard, 2005. http://www.blackboard.com/ Visited Nov. 2005.

9. WebCT, 2005. WebCT http://www.webct.com Visited Nov. 2005.

10. Ganesha, 2005. http://www.anemalab.org/ganesha/ Visited Nov. 2005.

11. COSE., 2003. http://www.staffs.ac.uk/COSE/ Visited Nov. 2005.

12. Djoudi, M., Harous S., 2002. An environment for cooperative learning over the internet. Intl. Conf. Artificial Intelligence (IC-AI'2002), Las Vegas, Nevada, USA, Jun. 24-27.

13. Khentout, C., Douidi, L., Djoudi M., 2002. Conception d'assistants pour l'enseignant et l'apprenant en enseignement sur le Web. 2eme Colloque international sur l'université virtuelle, Alger.

14. Quarteroni, P., 1996. Un hypermédia pédagogiquement efficace. Revue Educatechnologiques, Université Laval, Canada.

15. Rhéaume, J., 1997. Les hypertextes et les hypermédias. Revue Éducatechnologiques, Faculté des sciences de l'éducation, Université Laval, Canada.

16. Murray, T., 1998. Authoring knowledge-based tutors: Tools for content, instructional strategy, student model and interface design. J. Learning Sci., 7: 1.

17. Behaz A., Djoudi M., 2005. Génération dynamique de documents hypermédias adaptatifs dans un environnement numérique de travail. Revue africaine de la recherche en informatique et mathématiques appliquées ARIMA, pp. 25-53. 